\title{
A Genome Sequence Resource of Nigrospora sphaerica Causing Fruit Dried-Shrink Disease in Akebia trifoliata
}

\author{
Xiujing Hong, ${ }^{1,2,3}$ Shengxiong Huang, ${ }^{3}$ and Yongsheng Liu ${ }^{1,3,4, \dagger}$ \\ ${ }^{1}$ School of Horticulture, Anhui Agricultural University, Hefei, 230036, China \\ ${ }^{2}$ Key Laboratory of Genetic Improvement and Ecophysiology of Horticultural Crop, Anhui Province, \\ Anhui Academy of Agricultural Sciences, Hefei, 230001, China \\ ${ }^{3}$ School of Food and Biological Engineering, Hefei University of Technology, Hefei, 230009, China \\ ${ }^{4}$ Ministry of Education Key Laboratory for Bio-Resource and Eco-Environment, College of Life Sci- \\ ence, State Key Laboratory of Hydraulics and Mountain River Engineering, Sichuan University, \\ Chengdu, 610064, China
}

\begin{abstract}
Nigrospora sphaerica is a worldwide plant pathogen causing fruit or leaf diseases on a variety of plant hosts such as Citrullus lanatus, Vigna unguiculata, Hylocereus polyrhizus, and Akebia trifoliata and other potential hosts. Here we report the first genome resource with high-quality assembly of the $N$. sphaerica strain ZJJ-C1, which causes fruit driedshrink disease in A. trifoliata in China. The genome sequence of ZJJ-C1 will be useful for studying the evolution, host adaptation, and pathogenicity of $N$. sphaerica, which will be beneficial for a better understanding of the mechanisms of host-pathogen interaction during the endophytic period.
\end{abstract}

\section{Genome Announcement}

The genus Nigrospora includes several plant pathogens causing diseases on a variety of plant hosts. Nigrospora sphaerica is a damaging pathogen that causes leaf spots on Citrullus lanatus (Ismail and Abd Razak 2021), Vigna unguiculata (Deepika et al. 2021), Arachis hypogaea (Liu et al. 2020), and Parthenium hysterophorus (Zafri et al. 2021); it also causes fruit dried-shrink disease on Hylocereus polyrhizus (Kee et al. 2019) and Akebia trifoliata (Hong et al. 2021). In addition, $N$. aurantiaca and $N$. oryzae are the causal agents of leaf spot or wilt disease on Hibiscus mutabilis (Han et al. 2021), Nicotiana tabacum (Huang et al. 2021), and a number of other plant species.

N. sphaerica ZJJ-C1 was originally isolated from fruits of Akebia trifoliata at Zhangjiajie City, Hunan Province, China, and identified as a pathogen causing dried-shrink disease on fruits (Hong et al. 2021). In the current study, this isolate was repurified employing singlespore isolation approach. This isolate was grown in sterile potato dextrose agar (PDA) at $25^{\circ} \mathrm{C}$ for 7 days before mycelia were harvested and ground with liquid nitrogen. Highly purified genomic DNA was extracted using a Cell Culture DNA Midi Kit (cat. no. 13343, Qiagen, Valencia, CA, U.S.A.) according to the manufacturer's protocol. DNA quality and concentration were assessed using $0.5 \%$ agarose gel electrophoresis and via Qubit fluorometer and Nanodrop 2000 spectrophotometer (Thermo Fisher Scientific, Carlsbad, CA, U.S.A.). The A260/A280 ratio of 1.86 in the sample genomic DNA, and concentration of $163.0 \mathrm{ng} / \mu \mathrm{l}$ were recorded. Whole-genome sequencing data were obtained using the Oxford Nanopore Technology (ONT) PromethION platform (Oxford Nanopore, Oxford, UK) and short-read sequenced on a DNA Nano Ball Sequencing (DNBseq) platform.

${ }^{\dagger}$ Corresponding author: Y. S. Liu; liuyongsheng1122@ahau.edu.cn

The author(s) declare no conflict of interest.

Accepted for publication 25 September 2021.

\section{Funding}

This work was supported by grants from National Natural Science Foundation of China (31972474, 31671259, and 31440028), Leading Talent Group Funding of Anhui Province (WRMR-2020-75) and National Foundation for Germplasm Repository of Special Horticultural Crops in Central Mountain Areas of China (NJF2017-69).

\section{Keywords}

Nigrospora sphaerica, genome sequence resource, fruit dried-shrink disease, Akebia trifoliata 
Table 1. Genome statistics of Nigrospora sphaerica isolate ZJJ-C1

\begin{tabular}{lc} 
Features & Strain ZJJ_C1 \\
Genome size $(\mathrm{Mb})$ & 51.75 \\
BUSCO completeness (\%) & 99.3 \\
Number of contigs & 33 \\
N50 $(\mathrm{kb})$ & 4.64 \\
N90 $(\mathrm{kb})$ & 1.62 \\
GC content (\%) & 56.09 \\
Number of genes & 11,518 \\
Protein-coding genes & 10,106 \\
Repeat rate $(\%)$ & $16.87 \%$ \\
GenBank accession no. & JAGVVL000000000 \\
\hline
\end{tabular}

All the raw data of about $43.39 \mathrm{~Gb}$ were trimmed by SOAPnuke (version 1.5.6) (Chen et al. 2017) (https://github.com/BGl-flexlab/SOAPnuke). High-quality reads were de novo assembled using CANU (version 2.1) (Koren et al. 2017), Samtools (Li and Durbin 2009), GATK (version 3.5) (McKenna et al. 2010), and Racon (Vaser et al. 2017) software. The nuclear genome of $N$. sphaerica consists of 33 contigs with a total assembly length of $51,752,671$ bp (N50 = 4.64 Mbp), 56.09\% GC content. Genome completeness was assessed through the presence of conserved single-copy fungal genes using Benchmarking Universal Single-Copy Orthologs (BUSCO) (version 3.0) (Simão et al. 2015), and its BUSCO completeness was $99.3 \%$ (753/758). The completeness of the assembly about gene set was verified with BUSCO (version 3.0) (Simão et al. 2015) software, which is based on a set of 746 common fungal genes. About $98.4 \%$ (746/758) of the BUSCOs in full length genes were found in the assembly. Assembled contigs with lengths less than $300 \mathrm{bp}$ were discarded in the following analysis.

We constructed a de novo repeat library using Piler (Edgar and Myers 2005) (http:// www.drive5.com/piler/), RepeatScout (https://bix.ucsd.edu/repeatscout/), and RepeatModeler (http://www.repeatmasker.org/RepeatModeler/) software. The library was searched as the reference database, and repeats were predicted using RepeatMasker (version 4.1.0). A total of $8,728,703 \mathrm{bp}$ of repeat sequences that accounted for $16.87 \%$ of the assembly, including Retrotransposon, DNA transposon, Tandem Repeat sequence, and other transposon, were detected in the genome assembly. A summary of the genome assemblies and gene prediction is shown in Table 1.

To obtain functional information, the protein sequences were aligned against the NCBI nonredundant protein sequences (Nr) (https://ttp.ncbi.nlm.nih.gov/blast/db/FASTA/) database, Swiss-Prot (https://www.uniprot.org/uniprot/?query=reviewed:yes) database, Gene Ontology (GO) (http://geneontology.org/) database, the Kyoto Encyclopedia of Genes and Genomes (KEGG) database (https://www.genome.jp/kegg/), Clusters of Orthologous Groups (COG) database (https://www.ncbi.nlm.nih.gov/research/cog-project), Evolutionary Genealogy of Genes: Nonsupervised Orthologous Groups (eggNOG) (version 5.0.0) (http://eggnog5.embl. de/) database, and Protein family (Pfam) (http://pfam.xfam.org/) database by DIAMOND (Buchfink et al. 2015) with an e-value cutoff of $1 \mathrm{e}^{-5}$. The result of the functional annotation indicated that 10,096 (87.65\%), 7,220 (62.68\%), 3,107 (26.98\%), 4,001 (34.74\%), 8,313 (72.17\%), 8,313 (72.17\%), and 7,534 (65.41\%) of the genes were annotated in the database of $\mathrm{Nr}$, Swiss-Prot, GO, KEGG, COG, eggNOG, and Pfam, respectively. There are 10,106 genes with functional annotations, accounting for $87.74 \%$ of total predicted genes. Of the proteincoding gene models, $65.41 \%(7,535)$ contained PFAM domains annotated by InterProScan 5.2-45.0 (threshold e-value of $1 \mathrm{e}^{-5}$ ) (Ohm et al. 2012). Meanwhile 1,118 proteins were predicted with signal peptide by using SignalP v4.1 (Petersen et al. 2011). Among the 1,118 proteins, 908 were computationally predicted to localize in the extracellular space as secreted proteins based on results from WoLF PSort v0.2 (Horton et al. 2007). In addition, genes coding for the putative chitiase and hydrolase, the main proteases of fungal involving host invasion, such as NSph01G001130, NSph01G023940, NSph09G001450, NSph17G006420, and NSph01G001130, have been validated as genes derived from N. sphaerica genome by PCR amplification and Sanger-sequencing.

In summary, this study reports a high-quality genome sequence resource of $N$. sphaerica ZJJ-C1, a strain causing fruit dried-shrink disease of Akebia trifoliata in China, which will allow further analysis of species diversity and evolutionary mechanisms and may serve as a 
foundation for understanding the interacting mechanisms between plants and fungal pathogens.

The assembled whole genome of ZJJ-C1 has been deposited at GenBank under the accession number of JAGVVL000000000 (https://www.ncbi.nlm.nih.gov/nuccore/JAGVVL000000000.1/).

\section{Literature Cited}

Buchfink, B., Xie, C., and Huson, D. H. 2015. Fast and sensitive protein alignment using DIAMOND. Nat. Methods 12:59-60.

Chen, Y. X., Chen, Y. S., Shi, C. M., Huang, Z. B., Zhang, Y., Li, S. K., Li, Y., Ye, J., Yu, C., Li, Z., Zhang, X. Q., Wang, J., Yang, H. M., Fang, L., and Chen, Q. 2017. SOAPnuke: a MapReduce acceleration-supported software for integrated quality control and preprocessing of high-throughput sequencing data. Gigascience 7:gix120.

Deepika, Y. S., Mahadevakumar, S., Amruthesh, K. N., and Lakshmidevi, N. 2021. First report of Nigrospora sphaerica associated with leaf spot disease of cowpea (Vigna unguiculata) from India. Plant Dis. 105:506.

Edgar, R. C., and Myers, E. W. 2005. PILER: identification and classification of genomic repeats. Bioinformatics 21 (Suppl. 1):i152-i158.

Han, S., Yu, S. T., Zhu, T. H., Li, S. J., Qiao, T. M., Liu, Y. G., Lin, T. T., and Yong, C. L. 2021. Nigrospora oryzae causing black leaf spot disease of Hibiscus mutabilis in China. Plant Dis. 105:2255.

Hong, X. J., Chen, S. J., Wang, L., Liu, B., Yang, Y., Tang, X., Liu, Y. S., and Huang, S. X. 2021. First report of Nigrospora sphaerica causing fruit driedshrink disease in Akebia trifoliata from China. Plant Dis. 105:2244.

Horton, P., Park, K. J., Obayashi, T., Fujita, N., Harada, H., Adams-Collier, C. J., and Nakai, K. 2007. WoLF PSORT: protein localization predictor. Nucleic Acids Res. 35:W585-W587.

Huang, Y., Li, Z., Wang, H. C., Chen, Q. L., and Li, W. H. 2021. First report of leaf spot caused by Nigrospora aurantiaca in tobacco in China. Plant Dis. 105:1569.

Ismail, S. I., and Abd Razak, N. F. 2021. First report of Nigrospora sphaerica causing leaf spot on watermelon (Citrullus lanatus) in Malaysia. Plant Dis. 105:488.

Kee, Y. J., Hafifi, A. B. M., Huda-Shakirah, A. R., Wong, K. L., Jin, X. L., Nordahliawate, M. S. S., Zakaria, L., and Mohd, M. H. 2019. First report of reddish brown spot disease of red-fleshed dragon fruit (Hylocereus polyrhizus) caused by Nigrospora lacticolonia and Nigrospora sphaerica in Malaysia. Crop Prot. 122:165-170.
Koren, S., Walenz, B. P., Berlin, K., Miller, J. R., Bergman, N. H., and Phillippy, A. M. 2017. Canu: scalable and accurate long-read assembly via adaptive k-mer weighting and repeat separation. Genome Res. 27:722-736.

$\mathrm{Li}, \mathrm{H}$., and Durbin, R. 2009. Fast and accurate short read alignment with Burrows-Wheeler transform. Bioinformatics 25:1754-1760.

Liu, X. Y., Yu, F. Y., Fu, D. Q., and Yang, W. B. 2020. First report of leaf blight on peanut caused by Nigrospora sphaerica in China. J. Plant Pathol. 102: 1269.

McKenna, A., Hanna, M., Banks, E., Sivachenko, A., Cibulskis, K., Kernytsky, A., Garimella, K., Altshuler, D., Gabriel, S., Daly, M., and DePristo, M. A. 2010. The Genome Analysis Toolkit: A MapReduce framework for analyzing nextgeneration DNA sequencing data. Genome Res. 20:1297-1303.

Ohm, R. A., Feau, N., Henrissat, B., Schoch, C. L., Horwitz, B. A., Barry, K. W., Condon, B. J., Copeland, A. C., Dhillon, B., Glaser, F., Hesse, C. N., Kosti, I., LaButti, K., Lindquist, E. A., Lucas, S., Salamov, A. A., Bradshaw, R. E., Ciuffetti, L., Hamelin, R. C., Kema, G. H., Lawrence, C., Scott, J. A., Spatafora, J. W., Turgeon, B. G., de Wit, P. J., Zhong, S., Goodwin, S. B., and Grigoriev, I. V. 2012. Diverse lifestyles and strategies of plant pathogenesis encoded in the genomes of eighteen Dothideomycetes fungi. PLoS Pathog 8:e1003037.

Petersen, T. N., Brunak, S., von Heijne, G., and Nielsen, H. 2011. SignalP 4.0: discriminating signal peptides from transmembrane regions. Nat. Methods 8 : 785-786.

Simão, F. A., Waterhouse, R. M., Ioannidis, P., Kriventseva, E. V., and Zdobnov, E. M. 2015. BUSCO: assessing genome assembly and annotation completeness with single-copy orthologs. Bioinformatics 31:3210-3212.

Vaser, R., Sovic, I., Nagarajan, N., and Sikic, M. 2017. Fast and accurate de novo genome assembly from long uncorrected reads. Genome Res. 27:737746.

Zafri, A. S., Muhamad, R., Wahab, A., Mokhtar, A. S., and Mohd Hata, E. 2021. First report of leaf blight on Parthenium hysterophorus caused by Nigrospora sphaerica in Malaysia. Plant Dis. 105:3297. 